\title{
Terahertz Time-Domain Spectroscopy and Low- Frequency Raman Scattering of Crystalline and Glassy Pharmaceutical Indapamide
}

\author{
Yukiko Kobayashi ${ }^{1}$, Tomohiko Shibata ${ }^{2}$, Tatsuya Mori ${ }^{2}$, Seiji Kojima ${ }^{2, *}$ \\ ${ }^{1}$ School of Engineering Sciences, University of Tsukuba, Tsukuba, Japan \\ ${ }^{2}$ Graduate School of Pure and Applied Sciences, University of Tsukuba, Tsukuba, \\ Ibaraki 305-8573, Japan \\ *E-mail address: kojima@ims.tsukuba.ac.jp
}

\begin{abstract}
We performed terahertz time-domain spectroscopy and low-frequency Raman scattering on crystalline and glassy states of pharmaceutical indapamide (IND). We have determined the real and imaginary part of the complex dielectric constants and the imaginary part of the Raman susceptibility in the THz region. Several phonon peaks have been observed in the crystalline IND and the mutual exclusion principle of infrared and Raman spectroscopy holds in the measured frequency range. In a glassy state of IND, a broad absorption peak has been observed in both the THz and the Raman spectra with different spectral shape, and this disagreement indicates that the far-infrared and Raman lightvibration coupling constants are different in the glassy IND. A clear boson peak has been observed in the Raman spectra of the glassy IND at about $0.5 \mathrm{THz}$.
\end{abstract}

Keywords: Indapamide; terahertz time-domain spectroscopy; low-frequency Raman scattering

\section{INTRODUCTION}

Commercial type indapamide (IND) is a drug material used in the treatment of hypertension. It exerts spasmolytic effects on blood vessels, consequently reducing the blood pressure. It is an organic glass former with a relatively high glass transition temperature $T_{g}=$ $376 \mathrm{~K}$ [1]. In the crystalline state of commercial type IND, the unit cell contains four IND molecules and those molecules form two types of cavities. The cavity encapsulates water molecules in non-stoichiometric. The water molecules are weakly bounded in the cavity, and can easily come out and in from the IND framework [2,3].

Terahertz time-domain spectroscopy (THz-TDS) is a kind of the far-infrared (far-IR) spectroscopy and has attached much attention, because this technique enables us to determine a complex dielectric constant without the Kramers-Kronig transformation, which is often used for Fourier-transform IR spectroscopy. THz-TDS has been extensively utilized for various kinds of crystalline and glassy materials to investigate low-energy excitations such as ferroelectric soft modes, boson peak, superconducting gap, and the low-frequency anharmonic phonon modes [4-7].

THz-TDS is useful as a vibrational spectroscopy to elucidate not only intra-molecular vibration but also inter-molecular vibration of organic materials. Reflecting the structure of 
molecular and crystal, $\mathrm{THz}$ spectra show a characteristic shape of each material. Therefore, the $\mathrm{THz}$ frequency region is called as a fingerprint region, and as an example of application for security, THz-TDS is used for seeing through packaging materials to probe for the presence of the illicit drugs [8,9]. THz-TDS is also used for the study of pharmaceutical drugs and is effective to discriminate polymorphic, because several forms of polymorphic drugs show completely different $\mathrm{THz}$ spectra. In addition to crystalline polymorphism, amorphous polymorphism also attracts much attention from fundamental physics and application for drug detection [5,10-12].

In this study, to investigate the dynamical properties of crystalline and glassy pharmaceutical IND in the THz region, we performed THz-TDS and low-frequency Raman scattering measurements.

\section{EXPERIMENTAL}

Indapamide $\left(\mathrm{C}_{16} \mathrm{H}_{16} \mathrm{ClN}_{3} \mathrm{O}_{3} \mathrm{~S}\right)$ with the purity $>99 \%$ was purchased from SigmaAldrich Corp. Crystalline, and glassy samples were made by melt-quenching into pellets or thin plates. The thicknesses of the commercial type crystalline samples used for THz-TDS measurement were $0.739,1.437$ and $2.839 \mathrm{~mm}$ to obtain appropriate intensity of transmission spectra. The thickness of the glassy sample used for THz-TDS was $1.071 \mathrm{~mm}$. All samples were measured at room temperature (296K).

The present THz-TDS measurements were performed by conventional transmission configuration (RT-10000, Tochigi Nikon Corp.). Low-temperature grown GaAs photoconductive (PC) antennas were utilized for the THz pulse emitter and detector. The PC antennas were triggered by a mode-locked Ti:sapphire pulsed laser with a wave-length of 780 $\mathrm{nm}$, a pulse width of less than $100 \mathrm{fs}$, and a repetition rate of $80 \mathrm{MHz}$. The available frequency range is from 0.2 to $4.0 \mathrm{THz}$.

Figure 1(a) shows the measured time-domain $\mathrm{THz} E$-field waveforms transmitted through the air (reference) and IND with a thickness of $0.739 \mathrm{~mm}$. Figure 1(b) shows the frequency-domain power spectra. To calculate the frequency dependent complex dielectric constant $\hat{\varepsilon}=\varepsilon^{\prime}+i \cdot \varepsilon^{\prime \prime}$ from the measured time-domain waveforms, we have used following equation,

$$
\frac{E_{\text {sam }}(\omega)}{E_{r e f}(\omega)}=\frac{4 \hat{n}}{(\hat{n}+1)^{2}} \exp \left\{i \frac{(\hat{n}-1)}{c} \omega d\right\},
$$

where $E_{\text {sam }}(\omega)$ and $E_{\text {ref }}(\omega)$ are the amplitude spectra of THz pulse transmitted the sample and the reference, respectively. $\hat{n}, c$ and $d$ are the complex refractive index, the light velocity and a thickness of sample, respectively. Then, the complex dielectric constant is obtained from the relation of $\hat{\varepsilon}=\hat{n}^{2}$.

The depolarized Raman scattering spectra were measured in the frequency range from 0.1 to $3.5 \mathrm{THz}$ under a scattering angle $180^{\circ}$, using a single frequency green-YAG laser with wavelength $532 \mathrm{~nm}$. The spectrometer was a double-grating monochromator (U-1000, HORIBA Corp.) and the spectral resolution of the Raman spectrometer was $0.04 \mathrm{THz}$. All Raman spectra were measured at room temperature. The observed Raman intensity spectra $I_{\text {raw }}(\omega)$ were converted to the imaginary part of the Raman susceptibility $\chi^{\prime \prime}(\omega)$ by the following equation, 


$$
\chi^{\prime \prime}(\omega)=\frac{I_{\text {raw }}(\omega)}{n_{B}(\omega)+1},
$$

where $n_{B}(\omega)=\left(\exp \left(\hbar \omega / k_{B} T\right)\right)^{-1}$ is Bose-Einstein distribution function.
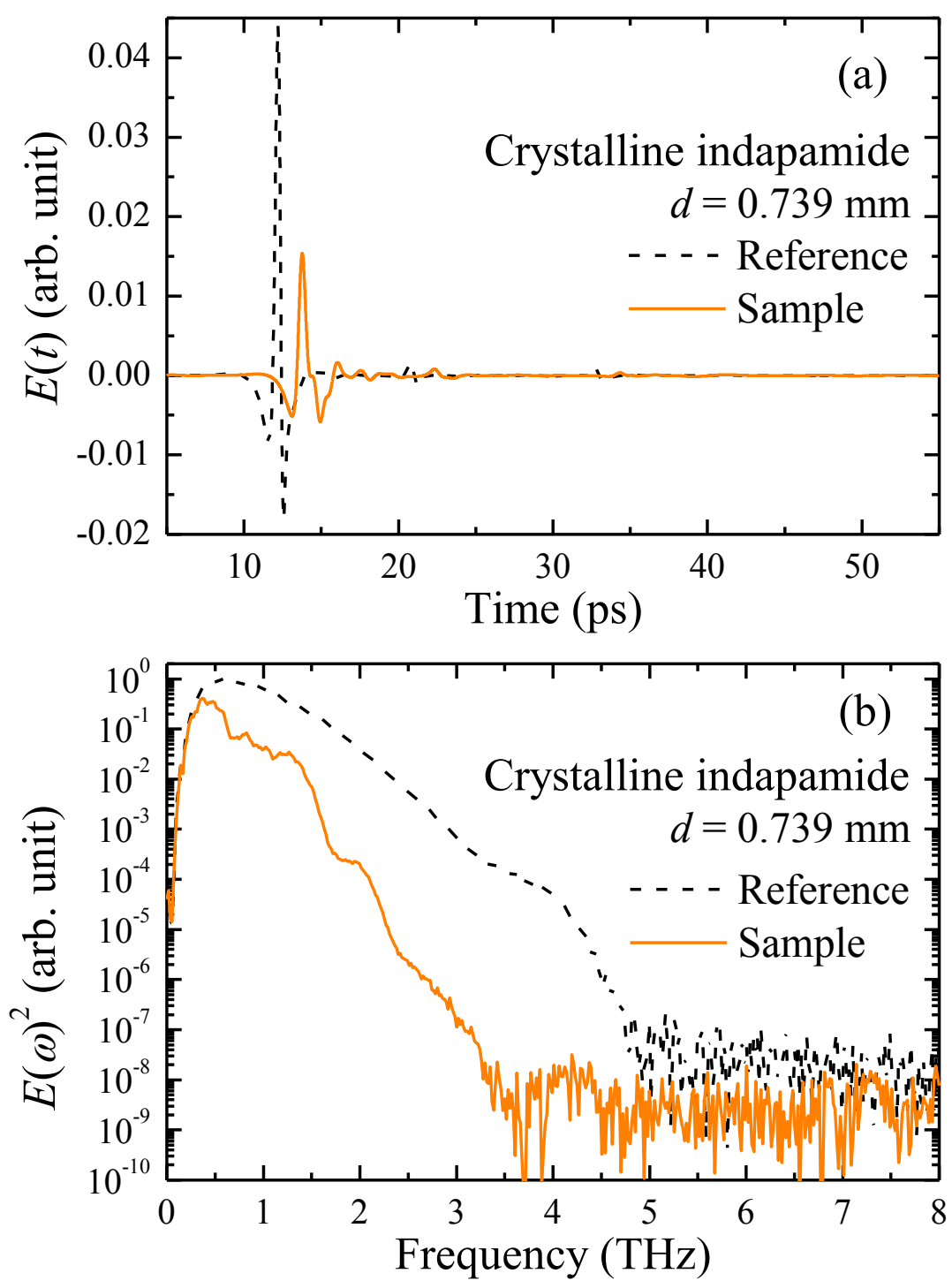

Figure 1. (a) Time-domain waveforms and (b) frequency-domain power spectra of reference and the commercial type crystalline state of IND at room temperature. 


\section{RESULTS \& DISCUSSION}

Figures 2(a) and 2(b) show the real and imaginary parts of the complex dielectric constants $\hat{\varepsilon}=\varepsilon^{\prime}+i \cdot \varepsilon^{\prime \prime}$ of the commercial type crystalline state of IND. For comparison, the imaginary part of the Raman susceptibility $\chi^{\prime \prime}(\omega)$ of the commercial type

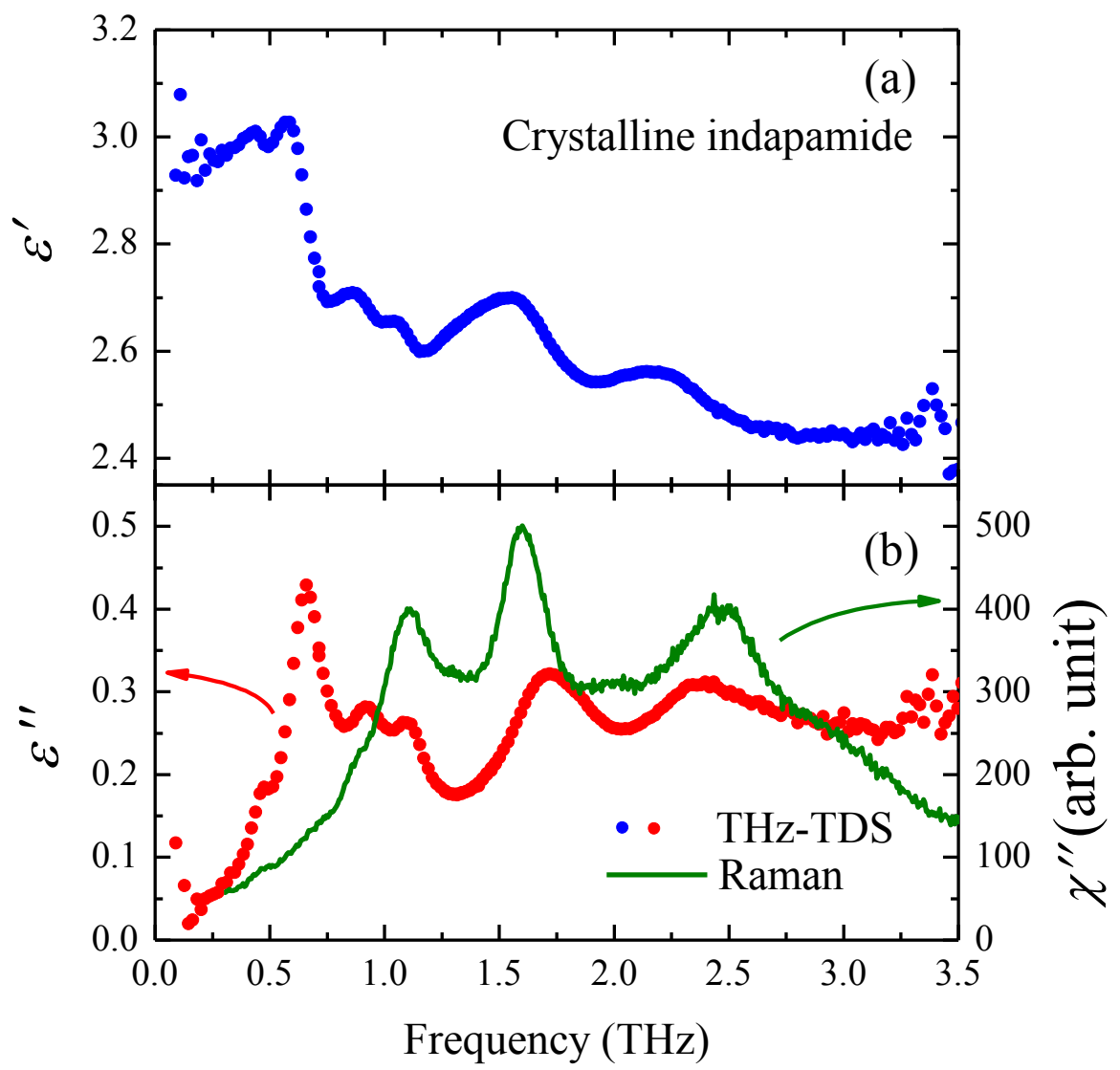

Figure 2. (a) The real part of the complex dielectric constant $\varepsilon^{\prime}(\omega)$ and (b) the comparison between the imaginary part of the complex dielectric constant $\varepsilon^{\prime \prime}(\omega)$ and the Raman susceptibility $\chi^{\prime \prime}(\omega)$ of the commercial type crystalline state of IND at room temperature.

Crystalline state of IND is also shown in Fig. 2(b). In the measured frequency range, several phonon peaks were observed, and those modes are attributed to the inter- or intramolecule vibrational modes. As shown in Fig. 2(b), the discrepancies of the phonon peak frequencies are clearly observed between $\varepsilon^{\prime \prime}(\omega)$ and $\chi^{\prime \prime}(\omega)$.

When the point group of a crystal structure has centro-symmetry, the mutual exclusion principle holds between Raman and IR activities. Some vibrational modes are only Raman active, and others are only IR active. Consequently, the crystalline IND has a centrosymmetry and this result is consistent with the X-ray diffraction study [3].

Figure 3(a) shows the real part of the complex dielectric constant $\varepsilon^{\prime}(\omega)$ of the glassy state of IND at room temperature. Figure 3(b) shows the comparison of the imaginary parts between the dielectric constant $\varepsilon^{\prime \prime}(\omega)$ and the Raman susceptibility $\chi^{\prime \prime}(\omega)$ of the glassy state of IND. The spectral shapes of both $\varepsilon^{\prime \prime}(\omega)$ and $\chi^{\prime \prime}(\omega)$ show a broad asymmetric absorption 
peak at about 0.7 and $1.2 \mathrm{THz}$, respectively. The observed structure of the broad absorption bands can be attributed to the vibrational mode with the distribution of mode frequency reflecting the distribution of bond lengths and bond angles of the glassy disordered structure.

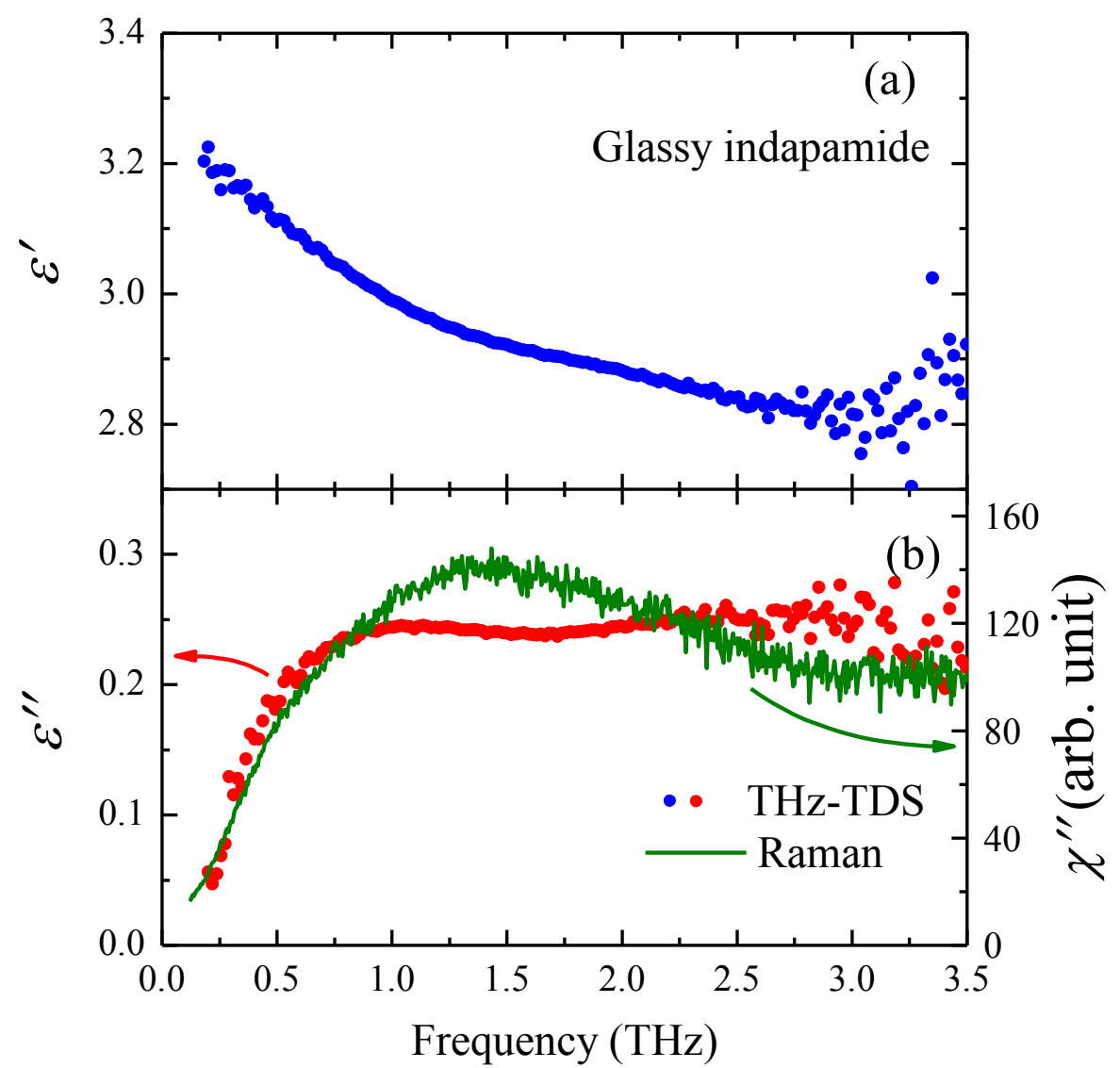

Figure 3. (a) The real part of the complex dielectric constants $\varepsilon^{\prime}(\omega)$ and (b) a comparison of the imaginary parts between the dielectric constant $\varepsilon^{\prime \prime}(\omega)$ and the Raman susceptibility $\chi^{\prime \prime}(\omega)$ of the glassy state of IND at room temperature.

In addition, $\varepsilon^{\prime \prime}(\omega)$ and $\chi^{\prime \prime}(\omega)$ of the glassy IND showed the obvious disagreement in their spectral shapes and peak positions each other. The obvious discrepancy in their spectral shapes and peak positions indicates that the far-infrared and Raman light-vibration coupling constants are different in the glassy IND. Furthermore, the spectral shapes of $\varepsilon^{\prime \prime}(\omega)$ and $\chi^{\prime \prime}(\omega)$ are reminiscent of the shape of the spectrum of each of the crystalline state shown in Fig. 2(b). Considering that the crystalline state of IND has a centro-symmetry, these experimental results might suggest that the IND has also the local centro-symmetry of nanoscale regions despite the macroscopic disordered glassy state, like a glassy state of pharmaceutical indomethacin [13].

The inelastic scattering spectra of glasses have generally shown a universal lowfrequency response called "boson peak" in the region below $3 \mathrm{THz}$ [14]. To obtain the clear boson peak, the reduced Raman intensity $\chi^{\prime \prime}(\omega) / \omega$ has been usually used [15]. Figure 4 shows the imaginary part of the complex dielectric constant and the Raman susceptibility divided by $\omega$ of glassy IND. A broad and asymmetric boson peak is clearly observed in the 
Raman spectra at about $0.5 \mathrm{THz}$, although a clear peak has not been observed in the $\mathrm{THz}$ spectra at the same frequency. The boson peak frequency in IND is lower than glycerol which is a typical hydrogen bonding organic material and has a boson peak at about $2 \mathrm{THz}$ [14].

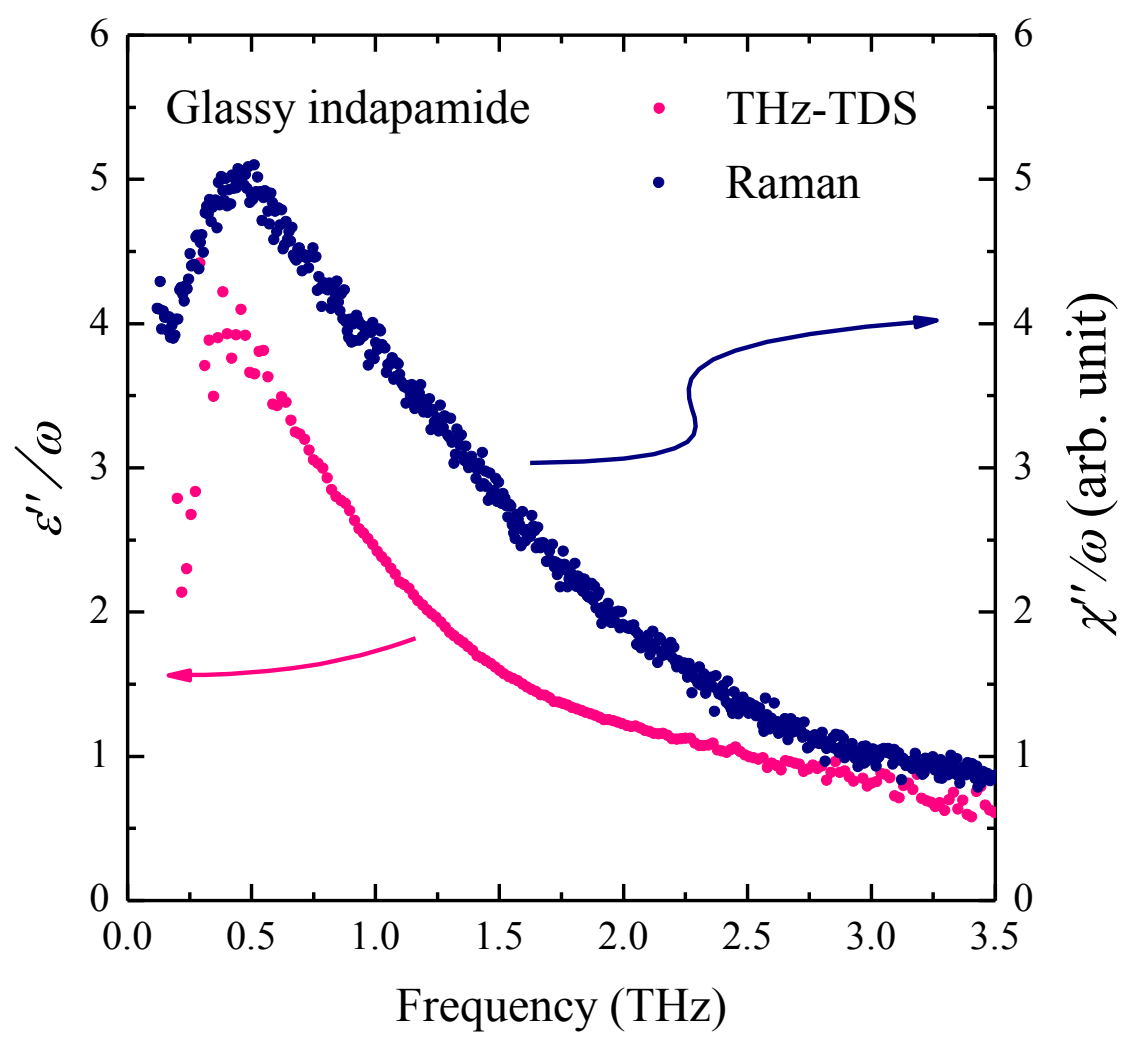

Figure 4. The imaginary part of the complex dielectric constant and the Raman susceptibility divided by $\omega$ of the glassy IND at room temperature.

\section{CONCLUSION}

We have investigated the terahertz dynamics of the crystalline and glassy states of pharmaceutical IND using THz-TDS and Raman spectroscopy at room temperature. Several phonon peak frequencies observed in the crystalline state showed discrepancies between THzTDS and Raman scattering measurements. It indicates that the crystal structure of the commercial type crystalline IND has a centro-symmetry. In a glassy state, $\varepsilon^{\prime \prime}(\omega)$ and $\chi^{\prime \prime}(\omega)$ showed a broad absorption band and a peak around $0.7 \mathrm{THz}$ and $1.2 \mathrm{THz}$, respectively. Both spectra showed different spectral shapes and the discrepancy indicates that the far-infrared and Raman light-vibration coupling constants are different in a glassy IND. A boson peak has been clearly observed in the Raman spectra at about $0.5 \mathrm{THz}$, although a peak has not been observed clearly in the $\mathrm{THz}$ spectra within the measured frequency range.

\section{Acknowledgement}

This work was partially supported by a Grants-in-Aid for Young Scientists (B) (24740194) from JSPS and the Sasakawa Scientific Research Grant from The Japan Science Society. 


\section{References}

[1] Z. Wojnarowska, K. Grzybowska, L. Hawelik, M. Dulski, R. Wrzalik, I. Gruszka, M. Paluch, Mol. Pharmaceutics 10 (2013) 3612.

[2] P. Ghugare, V. Dongre, P. Karmuse, R. Rana, D. Singh, A. Kumar, Z. Filmwala, Journal of Pharmaceutical and Biomedical Analysis 51 (2010) 532.

[3] M. Smrkolj, A. Meden, Pharmazie 61 (2006) 12.

[4] T. Mori, H. Igawa, S. Kojima, IOP Conf. Ser.: Mater. Sci. Eng. 54 (2014) 012006.

[5] S. Kojima, T. Shibata, H. Igawa, T. Mori, IOP Conf. Ser.: Mater. Sci. Eng. 54 (2014) 012001.

[6] T. Mori, K. Iwamoto, S. Kushibiki, H. Honda, H. Matsumoto, N. Toyota, Phys. Rev. Lett. 106 (2011) 015501.

[7] T. Mori, E. J. Nicol, S. Shiizuka, K. Kuniyasu, T. Nojima, N. Toyota, J. P. Carbotte, Phys. Rev. B 77 (2008) 174515.

[8] K. Kawase, Y. Ogawa, Y. Watanabe, H. Inoue, Opt. Express 11 (2013) 2549.

[9] J. Shen, G. Wang, D. Jiang, L. Liang, X. Xu, Optik 121 (2010) 1712.

[10] M. Otsuka, J. Nishizawa, N. Fukura, T. Sasaki, J Infrared Milli Terahz Waves 33 (2012) 953.

[11] P. Taday, I. Bradley, D. Arnone, M. Pepper, J. Pharm. Sci. 92 (2003) 831.

[12] C. Strachan, T. Rades, D. Newnham, K. Gordon, M. Pepper, P. Taday, Chem. Phys. Lett. 390 (2004) 20.

[13] C. Strachan, T. Rades, K. Gordon, JPP 59 (2007) 261.

[14] S. Kojima, Phys. Rev. B 47 (1993) 2924.

[15] T. Shibata, H. Igawa T. Kim, T. Mori, S. Kojima, J. Mol. Struct. 1062 (2014) 185. 